\title{
Surgical treatment of glomus jugulare tumors without rerouting of the facial nerve: an infralabyrinthine approach
}

\author{
Luis A. B. Borba, M.D., Samir Ale-Bark, M.D., and Charles London, M.D. \\ Department of Neurosurgery, Center for Neurological Surgery of Parana, Evangelical University \\ Medical School, Curitiba, Parana, Brazil
}

\begin{abstract}
Object. Glomus jugulare tumors are benign lesions located in the jugular foramen that may or may not extend into the middle ear, petrous apex, and upper neck; these growths sometimes invade intradurally. The surgical management of these tumors is a challenge to neurosurgeons and skull base surgeons. Because of their abundant vascularity, deep location, complex anatomy, and difficult surgical approach, their treatment, has been a controversial issue for many years. Despite advancements in nonsurgical techniques, the only treatment with proven efficacy is radical surgical removal. The authors present a series of patients treated with radical removal, in which the feasibility of removing glomus jugulare tumors with low morbidity and a surgical approach limited to tumor removal are discussed. The extent of surgical exposure is tailored with emphasis placed on the routine anterior transposition of the facial nerve.

Methods. Between May 1997 and March 2004, 24 patients with glomus jugulare tumors were treated; 17 patients were women and seven were men. Their mean age at the time of diagnosis was 50 years (range 18-71 years). The most common symptom was hearing loss in $77 \%$, followed by dysphagia and dysphonia in $55 \%$ of patients. In seven patients the clinical presentation was a facial palsy. Radical tumor removal was achieved in 23 patients. An anterior facial nerve transposition was not needed in any case. No surgery-related death was recorded in this series, although one patient died of a pulmonary embolism 70 days after the procedure. A one-stage procedure was performed in 23 patients and a two-stage procedure was used in the other patient. Cerebrospinal fluid leakage occurred in two patients. The lower cranial nerve function was worse in eight patients; however, only one had a new deficit. The facial nerve was preserved in all patients except one, in whom a large intradural tumor caused a temporary facial palsy. In the patients with preoperative facial palsy, the tumor only compressed the nerve in three and it invaded the nerve in four. The nerve was decompressed in the cases with no invasion and a graft was placed in the others. The greater auricular nerve was used as a graft in three and the sural nerve was used in one. On follow-up review, the facial nerve function was House-Brackmann Grade 3 in three patients and Grade 2 in three. After 6 months of follow up with no improvement, one patient was referred for a facial muscle transfer.

Conclusions. The surgical technique must be tailored to each case. The authors believe that the standard surgical approach to jugular foramen tumors with anterior transposition of the facial nerve should be avoided, and that the extent of surgical exposure must be tailored to each case based on the extent of the tumor and the clinical symptoms. Lower morbidity rates and radical removal can be achieved with a good surgical plan.
\end{abstract}

\section{KEY WORDS • glomus jugulare tumor • skull base tumor • paraganglioma • jugular foramen $\bullet$ infralabyrinthine approach}

Benign paragangliomas that originate in or near the jugular foramen are called glomus jugulare tumors. ${ }^{18}$ Their complex anatomy, abundant vascularity, and the critical relationship with the lower cranial nerves, ICA, jugular bulb, and facial nerve makes surgical removal of Fisch Class $\mathrm{C}$ and $\mathrm{D}$ glomus jugulare tumors a challenge for skull base surgeons. ${ }^{1-5}$

Abbreviations used in this paper: $\mathrm{CT}=$ computerized tomography; ECA = external carotid artery; ICA = internal CA; IJV = internal jugular vein; $\mathrm{MR}=$ magnetic resonance; $\mathrm{SCM}=$ sternocleidomastoid; VA = vertebral artery.
Rosenwasser ${ }^{18}$ was the first to attempt radical removal of a glomus jugulare tumor. In $1952 \mathrm{Capps}^{8}$ reviewed the current literature and added five new cases of glomus jugulare tumor. This author was the first surgeon to perform an anterior mobilization of the facial nerve, occlusion of the sigmoid sinus and the ligature of the IJV in the neck. In 1964, however, Shapiro and Neues ${ }^{20}$ were the first to describe the combination of mastoidectomy, anterior facial nerve transposition, and neck exposure with ligature of the IJV and arterial branches to the tumor. These authors pioneered the use of severe hypotension and hypothermia as crucial factors to avoid death caused by severe bleeding. 
In 1978 Fisch $^{10}$ restored this approach to its former position as state of the art in the management of glomus jugulare Class $\mathrm{C}$ tumors. The key element of the infratemporal approach described by Fisch is an anterior transposition of the facial nerve. This approach gives a straight, direct view of the jugular foramen, allowing a safe and wide exposure of the tumor. Despite very meticulous dissection of the facial nerve, a transitory weakness is often found in the postoperative period. ${ }^{11}$ During the last decade several articles have been published concerning radical surgery for removal of glomus jugulare tumors; however, few of them have addressed the need for facial nerve rerouting to attain radical removal of the mass.

In this report we present a series of 24 cases of glomus jugulare Class $\mathrm{C}$ and $\mathrm{D}$ tumors treated without standard rerouting of the facial nerve.

\section{CLINICAL MATERIAL AND METHODS}

\section{Patient Population}

Between May 1997 and March 2004, 24 patients harboring a Class $\mathrm{C}$ or $\mathrm{D}$ glomus jugulare tumor were treated at the Evangelical University Hospital of Parana, Brazil.

Of these 24 patients, 17 were women and seven were men. Their mean age at the time of diagnosis was 50 years; their ages ranged from 18 to 71 years. The most common symptom was hearing loss in $77 \%$, followed by dysphagia and dysphonia in $55 \%$ of patients. In seven patients the clinical presentation was facial palsy, and intradural tumor extension was found in four cases.

The patients were evaluated preoperatively with detailed neurological, otological, and laryngological examinations. Admission CT scans of the temporal bone and surrounding area were obtained, and MR images with and without contrast agents were acquired in all patients. The vascular anatomy and the blood supply to the tumor were demonstrated by cerebral angiograms, including a view of branches of the ECA. A postoperative CT scan was obtained in every case and an MR image was acquired 3 months after the surgical procedure.

\section{Surgical Approach}

The surgical approach was tailored to each patient, based on the extent of the tumor and the clinical symptoms. The patient is placed supine with the head turned $60^{\circ}$ to the opposite side and fixed in a three-point headrest. The ipsilateral shoulder is slightly elevated. The abdominal area and the lateral face of the thigh are also prepared so that fat and the fascia lata can be harvested.

A C-shaped incision is made: beginning in the midtemporal area, the incision runs posteriorly, slightly curving downward just behind the ear and the mastoid process. It then follows an anterior route, traversing the anterior border of the SCM muscle and ending at the level of the cricoid cartilage. The skin flap is reflected anteriorly. The external auditory canal is not transected. The greater auricular nerve and the external jugular vein are identified as they cross over the SCM muscle. The nerve is cut in its anterior portion close to the parotid gland and reflected posteriorly.

The posterior portion of the temporalis fascia is re- moved from the temporalis muscle by dissecting it posteriorly and inferiorly. The fascia remains connected to the insertion of the SCM muscle, which is detached from the mastoid process and rotated inferiorly and posteriorly. The neurovascular structures of the neck (the ICA, the ECA and its branches, and the (10th-12th cranial nerves) are dissected and exposed. The blood supply to the tumor, which generally originates from the ascending pharyngeal artery, the posterior auricular artery, and the occipital artery, is ligated. The posterior belly of the digastric muscle is detached and displaced anteriorly. An attempt to identify the facial nerve is made.

The next step of the procedure is the removal of the mastoid with exposure of the sigmoid sinus, labyrinth, and dura mater of the posterior fossa. The sigmoid sinus and jugular bulb are skeletonized. In patients with preoperative facial nerve palsy, the nerve is skeletonized in its vertical segment in the temporal bone and is exposed. In cases of tumor invasion, the facial nerve is cut and grafted to the greater auricular nerve. In situations of facial nerve compression without invasion by the tumor, the nerve is decompressed and kept in its original position.

The sigmoid sinus is ligated inferiorly as far as the mastoid emissary vein. A double suture is necessary to achieve total occlusion of the sinus. The IJV is isolated in the neck and is partially occluded. An attempt should be made to ligate the IJV at this stage because of the risk of tumor swelling and unacceptable bleeding.

Tumor removal begins by opening the sigmoid sinus and progressively exposing the jugular bulb, which is filled with the lesion. The tumor is removed by a combination of suction and coagulation. At this stage, the IJV can be totally occluded and opened. The bleeding in the jugular foramen area comes from the tumor itself, the inferior petrous sinus, and the condylar emissary vein, which must be plugged with Surgicel.

The preservation of the ninth, 10th, and 11th cranial nerves in the jugular foramen is a crucial matter. Preservation of the anterior wall of the IJV and the jugular bulb help protect the nerves. Extensive coagulation of the anterior wall of the jugular bulb must be avoided.

After affirming the removal of the extradural part of the tumor, the dura mater should be opened to search for intradural fragments. The dura mater may be grafted with fascia lata or the cavity completely filled with fat and glue. The posterior portion of the temporalis muscle is then rotated inferiorly and fixed to the SCM muscle. The muscle layers and the superficial planes are closed tightly (Fig. 1).

\section{RESULTS}

Admission CT scans and MR images depicted the tumor in all cases. Angiograms demonstrated a highly vascular mass that was supplied by branches of the ECA in all cases, and by the ICA and ECA in seven patients. Blood supply from branches of the VA was found in four patients.

Preoperative embolization of the tumor mass was performed in seven patients, by using particles in four and glue in three. All patients except one had a single tumor, and that one had multiple lesions consisting of vagal, carotid artery, and glomus jugulare tumors. Systemic arte- 


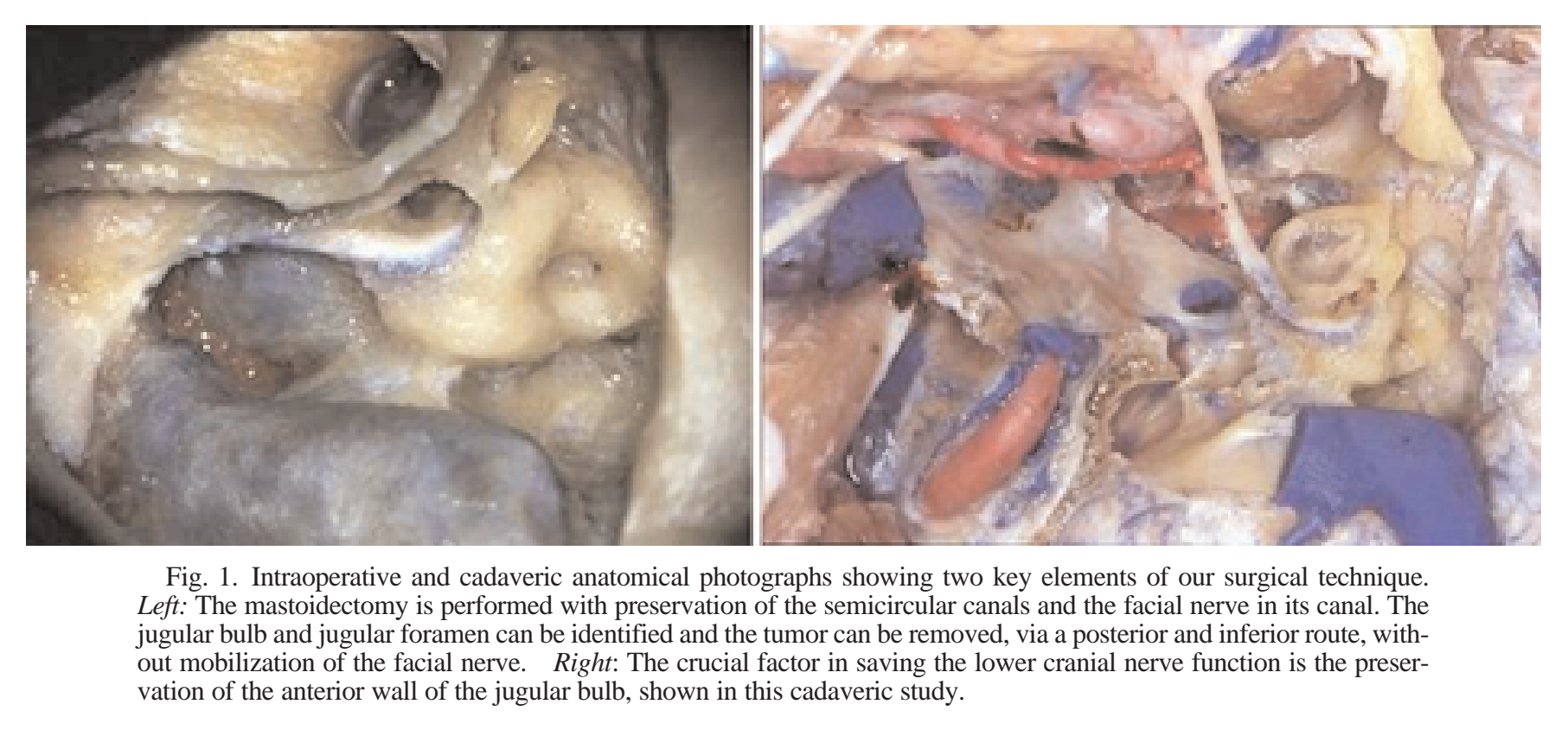

rial hypertension was diagnosed in 14 patients; however, none had a high serum catecholamine level or vanillylmandelic acid.

Twenty-six surgical procedures were performed in the 24 patients. A radical removal, defined as the absence of residual tumor on postoperative MR images, was achieved in 23 patients. In the other one radical removal was not possible because preoperative embolization with glue made the tumor bloody and hard, and it was surrounding the ICA. In one patient who had a multiple paraganglioma with vagal, carotid artery, and glomus jugulare tumors, a staged procedure was performed, essentially because of this 67-year-old woman's medical condition.

Cerebrospinal fluid leakage occurred in two patients treated only with lumbar drainage.

The facial nerve function was preserved in all patients except one; in this individual a large intradural tumor extension caused a temporary facial nerve palsy.

In the patients with preoperative facial nerve palsy, the tumor only compressed the nerve in three patients and invaded it in four. Decompression of the nerve was performed in the patients without tumor invasion and a graft was placed in the others. In the four patients in whom the facial nerve was invaded by tumor, a graft with the greater auricular nerve was performed in three and with the sural nerve in the other. On follow-up review, facial nerve function was House-Brackmann Grade 3 in three patients and Grade 2 in three patients. After 6 months of follow up with no improvement, one patient was referred by another physician for a facial muscle transfer.

Lower cranial nerve function was worse than before the operation in eight patients; however, only one had a new deficit. A tracheotomy was indicated in one case. Medialization of the vocal cord was performed in two cases.

One patient, a 67-year-old woman with severe heart and pulmonary disease caused by chronic high blood pressure, died during the follow-up period. She died of a pulmonary embolism 70 days after the surgical procedure.
At a mean follow-up period of 45 months, no patient had signs of recurrence, except one in whom the tumor was very aggressive, despite the typical pathological findings. This patient had metastasis and recurrence in the surgical field, and radiation therapy was indicated in this case (Fig. 2).

\section{DISCUSSION}

The temporal bone houses the facial nerve, vestibular and cochlear structures, and the ICA, and it marks the bone edge of the jugular foramen, along with the lower cranial nerves (ninth-11th) and the jugular bulb. Since 1978, when Fisch ${ }^{10}$ refined the surgical treatment of glomus jugulare tumors, the feasibility of surgical removal of these tumors has been advocated, despite the fact that a Class D2 lesion requires a two-stage surgery and a Class D3 is considered to be an inoperable tumor. ${ }^{11}$

With the refinement of surgical techniques in the last decade and increased interest among neurosurgeons, combined with better understanding of the surgical anatomy of the jugular foramen and temporal bone, it has become possible to remove a giant glomus jugulare tumor with good results and low morbidity. ${ }^{2,3}$

The glomus jugulare tumor is a benign lesion that rarely exhibits rapid growth; however, the morbidity related to the disease is well documented. Brown ${ }^{7}$ reported that $35 \%$ of patients with glomus jugulare tumors die within the 10year follow-up period.

Radiation therapy is associated with an increase in "stromal" cells, and a decrease in tumor vascularity and growth. Nevertheless, radiation therapy does not affect the "chief" cells, which keep the tumor alive., ${ }^{714}$ Recently management with embolization alone has been attempted, but there has been no evidence of its long-term efficacy. Based on these factors, the only treatment with recognized efficacy in the management of glomus jugulare tumors is radical gross-total removal. 


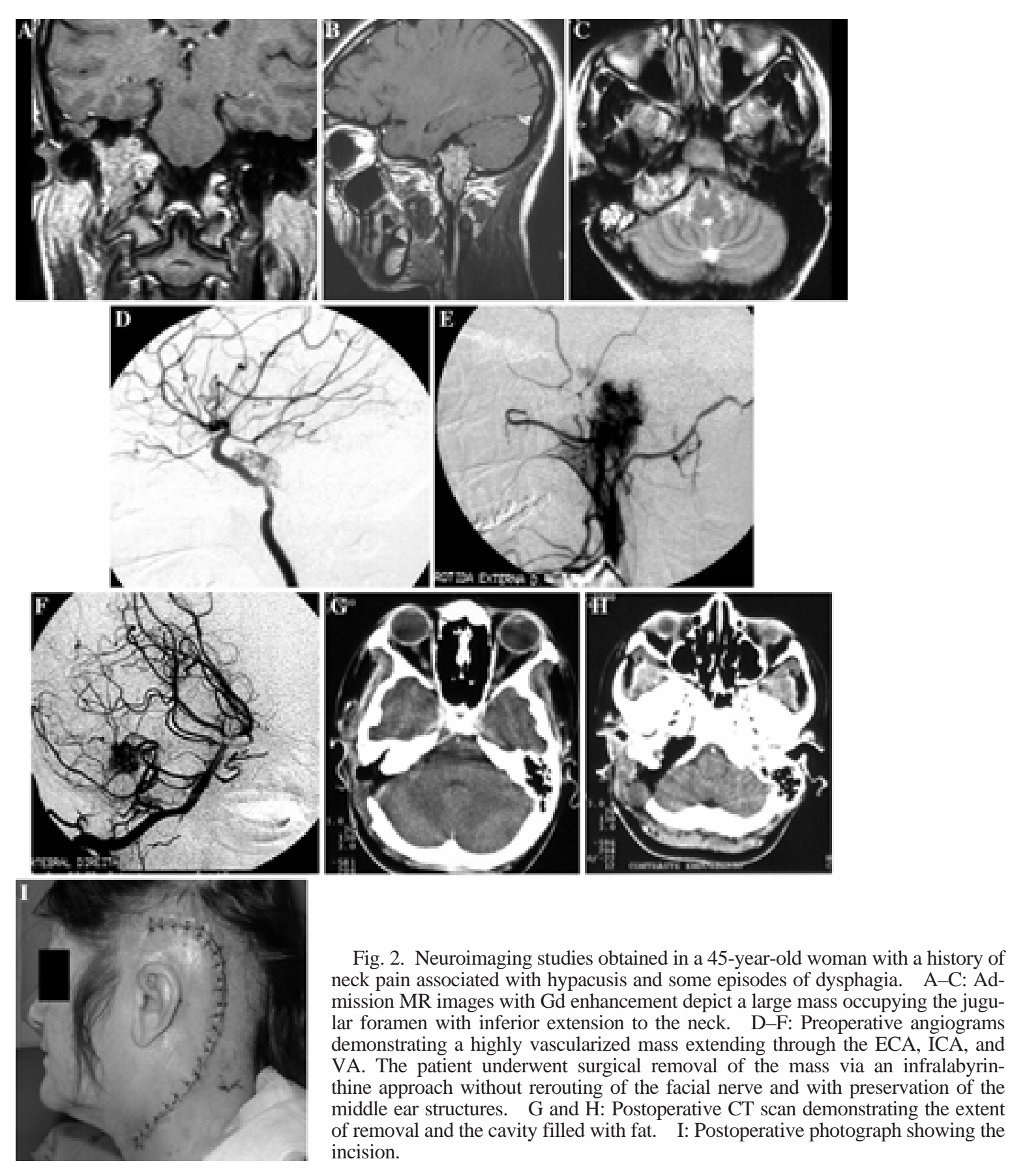

Lower cranial nerve function is the main source of morbidity and this aspect was well addressed by Al-Mefty and Teixeira. ${ }^{2}$ The preservation of the anterior wall of the jugular bulb is essential to save the nerves and their vascularization. In our experience, in situations in which the lower cranial nerve function is lost in the preoperative period, the surgical findings demonstrate invasion of the anterior wall of the jugular bulb by the tumor. ${ }^{6,17,19}$ In cases of normal function, overpacking or excessive coagulation must be avoided. ${ }^{6}$

Despite the outstanding work of Fisch and others, the surgical procedure has always been considered to be very high risk and has been associated with a high morbidity rate. Regardless of the expertise of the surgeon, the surgical approach by itself is associated with morbidity. ${ }^{15,16}$ Facial nerve weakness caused by anterior transposition of the facial nerve and conductive hearing loss are the rule and are directly related to the approach. ${ }^{11,17,18}$ In some situations the exposure is more than is really needed, and this gives rise to morbidity.

George, et al., ${ }^{12}$ have described a juxtacondylar approach to reach lesions located in the jugular foramen without removal of the petrous bone. In this approach the VA is displaced posteriorly and inferiorly, exposing the at- 
lantooccipital joint, which is partially removed, exposing the inferior pole of the jugular foramen. The IJV and jugular foramen are approached via an anterior and inferior route. This approach is limited to lesions located in the neck and jugular bulb, and it can be used to reach any superior extension of the tumor. In this situation these authors added a petrous removal with or without facial nerve transposition.

Green, et al., ${ }^{13}$ described a surgical technique in which the inferior portion of the vertical segment of the facial nerve is displaced anteriorly along with its attached blood supply through the stylomastoid artery, decreasing the incidence of postoperative facial weakness.

Our technique is similar to the intrabulbar dissection described by Al-Mefty and Teixeira, ${ }^{2}$ differing only in the extent of bone removal and the amount of facial nerve exposure. In our series, the surgical approach was tailored to each case, based on the extent of the tumor and the clinical symptoms. In patients in whom the clinical presentation consisted of lower cranial nerve deficits with the facial nerve function and hearing preserved, no attempt was made to expose the facial nerve, either in the canal or in the extratemporal portion. The labyrinth and middle ear structures are kept intact and the external auditory canal is not transected. In situations, in which the facial nerve is damaged by the tumor, the vertical and extratemporal segment of the facial nerve are exposed and dissected away from the lesion or grafted with greater auricular nerve. In situations in which the facial nerve and hearing function are lost, a total petrosectomy with closure of the external auditory canal is performed.

An infralabyrinthine approach, without opening of the middle ear or facial nerve transposition or exposure, allows exploration of tumors located in the jugular foramen with or without intradural extension. Nevertheless, in cases in which the ICA is encased by the tumor, we prefer to extend the dissection anteriorly, closing the external auditory canal and drilling the anterior wall of the tympanic cavity, thus exposing the ICA. The facial nerve is kept in its anatomical position and the labyrinth is also preserved.

The infralabyrinthine approach without rerouting of the facial nerve allows exposure of the entire tumor bed, and tumor removal can be accomplished safely. The only blind areas encountered with this approach are the portion under the facial nerve, which can be reached with a curved dissector, and the petrous apex, which can be reached with a small curette. Any bleeding from these areas can be easily controlled with gentle packing.

\section{CONCLUSIONS}

We believe that the standard surgical approach to jugular foramen tumors in which anterior transposition of the facial nerve is used should be avoided, and that the extent of surgical exposure must be tailored to each case, based on the extent of the tumor and the clinical symptoms. Lower morbidity and radical removal can be achieved with the aid of a good surgical plan.

\section{References}

1. Al-Mefty O, Fox JL, Rifai A, et al: A combined infratemporal and posterior fossa approach for the removal of giant glomus tumors and chondrosarcomas. Surg Neurol 28:423-431, 1987

2. Al-Mefty O, Teixeira A: Complex tumors of the glomus jugulare: criteria, treatment, and outcome. J Neurosurg 97: 1356-1366, 2002

3. Anand VK, Leonetti JP, Al-Mefty O: Neurovascular considerations in surgery of glomus tumors with intracranial extensions. Laryngoscope 103:722-728, 1993

4. Borba LA, Al-Mefty O: Intravagal paragangliomas: report of four cases. Neurosurgery 38:569-575, 1996

5. Borba LAB, Al-Mefty O: Paragangliomas of the skull base. Neurosurg Q 5:256-277, 1995

6. Borba LAB, Isolan G, Gomes M, et al: Tratamento cirúrgico dos tumores glômicos do forâmen jugular. Aspectos cirúrgicos e anatômicos. J Bras Neurocirurg 12:5-15, 2001

7. Brown JS: Glomus jugulare tumors revisited: a ten-year statistical follow-up of 231 cases. Laryngoscope 95:284-288, 1985

8. Capps FCW: Glomus jugulare tumours of the middle ear. $\mathbf{J}$ Laryngol Otol 66:302-314, 1952

9. Cole JM, BeilerD: Long-term results of treatment for glomus jugulare and glomus vagale tumors with radiotherapy. Laryngoscope 104:1461-1465, 1994

10. Fisch U: Infratemporal fossa approach to tumors of the temporal bone and base of the skull. J Laryngol Otol 92:949-967, 1978

11. Fisch U, Fagan P, Valavanis A: The infratemporal fossa approach for the lateral skull base. Otolaryngol Clin North Am 17:513-552, 1984

12. George B, Lot G, Tran Ba Huy P: The juxtacondylar approach to the jugular foramen (without petrous bone drilling). Surg Neurol 44:279-284, 1995

13. Green JD Jr, Brackmann DE, Nguyen CD, et al: Surgical management of previously untreated glomus jugulare tumors. Laryngoscope 104:917-921, 1994

14. Hawthorne MR, Makek MS, Harris JP, et al: The histopathological and clinical features of irradiated and nonirradiated temporal paragangliomas. Laryngoscope 98:325-331, 1988

15. Jackson CG: Basic surgical principles of neurotologic skull base surgery. Laryngoscope 103 (Suppl 60):29-44, 1993

16. Jackson CG: Neurotologic skull base surgery for glomus tumors. Diagnosis for treatment planning and treatment options. Laryngoscope 103 (Suppl 60): 17-22, 1993

17. Makek M, Franklin DJ, Zhao JC, et al: Neural infiltration of glomus temporale tumors. Am J Otol 11:1-5, 1990

18. Rosenwasser H: Carotid body tumor of the middle ear and mastoid. Arch Otolaryngol 41:64-67, 1945

19. Sen C, Hague K, Kacchara R, et al: Jugular foramen: microscopic anatomic features and implications for neural preservation with reference to glomus tumors involving the temporal bone. Neurosurgery 48:838-848, 2001

20. Shapiro MJ, Neues DK: Technique for removal of glomus jugulare tumors. Arch Otolaryngol 79:219-224, 1964

\footnotetext{
Manuscript received June 15, 2004.

Accepted in final form July 15, 2004

Address reprint requests to: Luis A. B. Borba, M.D., Rua Julia da Costa 1471-11, Curitiba, Parana, Brazil, CEP 80430-070. email: LuisABBorba@aol.com.
} 